\section{Reduction Mammaplasty is not Associated with a Decrease in BMI for Overweight or Obese Women}

\section{Abstract}

Introduction: Reduction mammaplasty can be a life-changing event for women with symptomatic macromastia. Many women experience improvement in physical symptoms, including resolution of neck, back and shoulder pain. There are other reported positive changes, including more physical activity, improved glucose control and a self-confidence. Adolescents have demonstrated weight loss following reduction mammaplasty but there is little objective data in the adult population.

Methods: After obtaining institutional review board approval, eligible patients were identified in our institution's electronic record database, eRecord (Epic; Madison, WI). Eligible subjects were identified using i2b2 software for the diagnosis of macromastia (ICD-9 611.1) and the CPT code for reduction mammaplasty (19318). A retrospective study chart review was performed from June 1, 2011 to time September 30, 2015 for97 women. The mean BMI was calculated at different time intervals post-operatively, including 1 week, 1 month, 3 months, 6 months, 12 months, 1 year, 2 years and $2+$ years. The BMI values for each patient were then compared to the 1 week postoperative BMI. The change was then analyzed to determine if there was any significant weight loss.

Results: There is a slight increase in BMI with increasing post-operative time (mean of 31.934 at one week compared to $33.2552+$ years) although this is not statistically significant. The lowest $50 \%$ of patients based on baseline-BMI was compared with the highest $50 \%$ of patients; each group showing no significant difference over time. Similar analyses using a mixed-effects model were performed given the longitudinal nature of the data, however this also showed that there was no significant change.

Conclusion: Despite well-document physical and psychological improvements following reduction mammaplasty, there is no statistically significant BMI decrease for women of all BMIs at time points from 1 month to more than 2 years when compared to their 1- week post-op BMI.

Keywords: Mammaplasty; Adolescents; Diagnosis; Psychological

\section{Paige Myers and Jose Guillherme Christiano \\ University of Rochester Medical Center, Rochester, NY 14642, USA}

\section{Corresponding author: Paige Myers \\ Đ paige_myers@urmc.rochester.edu}

Plastic Surgery Resident, University of Rochester Medical Center, Rochester, NY 14642, USA.

Tel: 315-286-3481

Citation: Myers P, Christiano JG. Reduction Mammaplasty is not Associated with a Decrease in BMI for Overweight or Obese Women. J Aesthet Reconstr Surg. 2017, 3:1.

Received: January 26, 2016; Accepted: February 07, 2017; Published: February 20, 2017

\section{Introduction}

Reduction mammaplasty can be a life-changing event for women with symptomatic macromastia. Many patients experience after surgery improvement or resolution of physical symptoms and other preoperative complaints, including neck, back and shoulder pain, intertrigo and shoulder grooving [1-5]. There is also evidence that reduction mammaplasty may result in correction of vertebral abnormalities [6,7]. Women presenting with macromastia are often less healthy, engage in less physical activity, and exhibit lower levels of self-esteem when compared to age-matched controls without the diagnosis [3]. This could set the stage for postoperative benefits beyond physical pain relief. In fact, other authors have reported increase in physical 
activity, improvement in glucose control with decreased insulin resistance, and increase in level of self-confidence [1-9]. The goal of our study was to determine whether reduction mammaplasty was associated with a drop in BMI in macromastia patients.

\section{Methods}

Approval for retrospective chart review was obtained from our institutional review board (RSRB 00059571). Eligible patients were identified in our institution's electronic record database, eRecord (Epic; Madison, WI), using i2b2 software (Partners Health Care; Boston, MA) for querying. All women who underwent bilateral reduction mammaplasty for macromastia between June of 2011 (implementation of eRecord in our institution) and September of 2015 were included. They were identified by searching for the diagnosis of macromastia (ICD-9 611.1) under Encounter Diagnoses, Past Medical History, or Problem List, and the CPT code for reduction mammaplasty (19318) under Procedures. Women were excluded from analysis if they were under the age of 18 , if they became pregnant, or were diagnosed with malignancy within the study period, or if there was insufficient documentation in the electronic record database.

The charts of ninety-seven patients who met our study's inclusion criteria were reviewed. The mean BMI was calculated for all 97 patients included in the study at different time intervals postoperatively, including 1 week, 1 month, 3 months, 6 months, 12 months, 1 year, 2 years and greater than two years. The BMI values for each patient at the aforementioned time intervals were then compared to the 1 week post-operative BMI (to account for the weight of the tissue removed). This change in $B M I$ was then analyzed to determine if there was any significant change. All analyses were carried out using SAS/STAT v9.4 (SAS Institute Inc.; Cary, NC).

\section{Results}

For preliminary exploration, the change in mean BMI was examined at each follow-up time, using 1-week post-operative
$\mathrm{BMI}$ as the baseline and following through 2 years and greater post-operative BMI. There is a slight increase in BMI with increasing post-operative time (mean of 31.934 at one week post-operative compared to $33.2552+$ years post-operative) although this relationship is not significant (Table 1).

To investigate this relationship further, the time trend of the lowest $50 \%$ of patients based on baseline-BMI was compared with the highest $50 \%$ of patients based on BMI. Looking at patients who comprise the lower $50 \%$ of baseline BMls, it is apparent that through three months after surgery, the average $\mathrm{BMI}$ is relatively maintained and unchanging. This is followed by an increase in BMI after three months post-operatively. There is large variability in these estimates, as illustrated in the $95 \%$ confidence interval bands, indicating no significant difference over time (Table $\mathbf{2}$ ).

For patients who comprise the higher $50 \%$ of baseline BMIs, there is an increase in BMI over time, with a slight decrease at six months after surgery (Table 3). Note that as more time elapses from date of the operation, there is less BMI information for fewer patients. In particular, the fewest observations at later time points are for patients who originally were in the lowest $50 \%$ of BMls. Thus, when looking at later time periods, most of the subjects examined already started with a higher BMI. As a result, overall summary results to also appear higher than what may have been the case if all BMI measurements were observed. Additionally, by twelve months after surgery, there is only a total of 37 observations -16 of which were from the lower $50 \%$ and 21 were from the higher $50 \%$-compared to the 97 observations at one week after surgery. With such small samples sizes, there is no significant change in BMI over time.

Since the data is longitudinal in structure, with patients being followed over time, a mixed-effects model with independent correlation structure was employed to incorporate the correlated nature of the data. This analysis was restricted to include BMI observations up through and including twelve months post-

Table 1: Mean BMI from 1 week post-op to +2 years post-op.

\begin{tabular}{|c|c|c|c|c|c|c|}
\hline Time (post-op) & N & N Miss & Mean & Std Dev & Lower 95\% CL for Mean & Upper 95\% CL for Mean \\
\hline 1 week & 97 & 0 & 31.9337073 & 5.8901688 & 30.7465755 & 33.1208392 \\
\hline month & 85 & 12 & 31.9928383 & 5.8646335 & 30.7278662 & 33.2578104 \\
\hline months & 73 & 24 & 32.4581355 & 6.3609511 & 30.9740158 & 33.9422552 \\
\hline months & 56 & 41 & 32.5591754 & 5.5282797 & 31.0786925 & 34.0396584 \\
\hline 2 months & 42 & 55 & 33.3060786 & 5.8668998 & 31.4778240 & 35.1343333 \\
\hline years & 42 & 55 & 33.0400511 & 6.2180927 & 31.1023570 & 34.9777452 \\
\hline
\end{tabular}

Table 2: Trend of mean BMI from 1 week post-op to +2 years post-op, lower 50\% BMI category.

\begin{tabular}{|c|c|c|c|c|c|c|c|}
\hline Time of Visit (post-operative) & N Obs & $\mathbf{N}$ & N Miss & Mean & Std Dev & Lower 95\% CL for Mean & Upper $95 \%$ CL for Mean \\
\hline 1 week & 49 & 49 & 0 & 27.4588956 & 2.5256041 & 26.7334574 & 28.1843338 \\
\hline 1 month & 49 & 43 & 6 & 27.4041576 & 2.4996681 & 26.6348733 & 28.1734419 \\
\hline 3 months & 49 & 35 & 14 & 27.4854026 & 2.8609938 & 26.5026168 & 28.4681883 \\
\hline 6 months & 49 & 25 & 24 & 27.9292590 & 3.1596298 & 26.6250279 & 29.2334900 \\
\hline 12 months & 49 & 18 & 31 & 28.1025020 & 3.0719489 & 26.5748578 & 29.6301463 \\
\hline 2 years & 49 & 20 & 29 & 28.4694471 & 3.0051369 & 27.0629997 & 29.8758944 \\
\hline $2+$ years & 49 & 16 & 33 & 28.5031039 & 3.1541137 & 26.8223953 & 30.1838125 \\
\hline
\end{tabular}


operatively to alleviate some issues with missing data at later time periods. Although again, there is no significance in this study $(p=0.5656)$. These analyses also indicate that no significant change occurs in BMI over time. In fact, there is no significant pairwise difference between any two time points (Table 4) Examining each of the BMI groups (i.e., lower $50 \%$ and upper $50 \%)$ separately, similar results of no significant change in $\mathrm{BMI}$ over time were discovered (Tables 5 and 6 ).

\section{Discussion}

Many other authors have reported physical and psychological improvements following reduction mammoplasty [1-9]. However, we found no statistically significant decrease in BMI following breast reduction in women of all included BMIs at any time interval. At 1 month, the mean BMI was 31.9928, compared to 31.9337 at one week, which is not significant. Similarly, at 3 months (BMI 32.4581), 6 months (BMI 32.5591), 1 year (BMI 33.3060), 2 years (BMI 33.0401) and more than 2 years (BMI 33.2545) there was no statistically significant change in BMI compared with 1 week post-op (BMI 31.9337) (Table 1). Clinically, there was a slight increase in BMI, although this was not statistically significant (mean of 31.9928 at one week postoperative compared to 33.0401) greater than 2+ years postoperatively. Given the correlated nature of the data following patients over a period of time, all intervals were compared to assess if a relationship exists with $\mathrm{BMI}$, however there was no pairwise significance in this study $(p=0.5656)$.

Similar results have been reported by Pauzenberger et al. [10]. In this study, only 50 women were retrospectively studied. It was discovered that 26 subjects gained weight and 18 lost weight, while 6 remained stable. The average weight loss was $3.5 \mathrm{~kg}$ (7.72 pounds) and the average weight gain was $4.5 \mathrm{~kg}$ (9.92 pounds). Neither weight gain nor loss was statistically significant.

Pike et al. studied adolescents undergoing breast reduction and also found no significant difference between mean pre-operative and post-operative BMIs overall, although overweight and obese patients progressed with significant weight gain post-operatively

Table 3: Trend of mean BMI from 1 week post-op to +2 years post-op, upper $50 \%$ BMI category.

\begin{tabular}{|c|c|c|c|c|c|c|c|c|}
\hline Time of Visit (post-operative) & N Obs & N & N Miss & Mean & Std Dev & Lower 95\% CL for Mean & Upper 95\% CL for Mean \\
\hline 1 week & 48 & 48 & 0 & 36.5017443 & 4.7077772 & 35.1347483 & 37.8687403 \\
\hline 1 month & 48 & 42 & 6 & 36.6907733 & 4.4014602 & 35.3191818 & 38.0623649 \\
\hline 3 months & 48 & 38 & 10 & 37.0382843 & 5.1262575 & 35.3533254 & 38.7232432 \\
\hline 6 months & 48 & 31 & 17 & 36.2929790 & 3.9716571 & 34.8361634 & 37.7497946 \\
\hline 12 months & 48 & 24 & 24 & 37.2087611 & 4.1592336 & 35.4524708 & 38.9650514 \\
\hline 2 years & 48 & 22 & 26 & 37.1951457 & 5.4161635 & 34.7937534 & 39.5965381 \\
\hline 2 years & 48 & 21 & 27 & 36.8747012 & 4.2825323 & 34.9253159 & 38.8240865 \\
\hline
\end{tabular}

Table 4: Pairwise comparison of post-operative BMI at all-time points post-operatively.

\begin{tabular}{|c|c|c|c|c|c|c|}
\hline & Time of Visit (post-op) & Estimate & Standard Error & DF & t Value & $\operatorname{Pr}>|t|$ \\
\hline 1 month & 1 week & 0.04470 & 0.1824 & 251 & 0.25 & 0.8065 \\
\hline 1 month & 12 months & -0.1760 & 0.2427 & 251 & -0.73 & 0.4690 \\
\hline 1 month & 3 months & -0.2184 & 0.1993 & 251 & -1.10 & 0.2741 \\
\hline 1 month & 6 months & -0.2145 & 0.2184 & 251 & -0.98 & 0.3269 \\
\hline 1 week & 12 months & -0.2207 & 0.2374 & 251 & -0.93 & 0.3534 \\
\hline 1 week & 3 months & -0.2631 & 0.1929 & 251 & -1.36 & 0.1739 \\
\hline 1 week & 6 months & -0.2592 & 0.2141 & 251 & -1.21 & 0.2271 \\
\hline 12 months & 3 months & -0.04244 & 0.2466 & 251 & -0.17 & 0.8635 \\
\hline 12 months & 6 months & -0.03854 & 0.2581 & 251 & -0.15 & 0.8814 \\
\hline 3 months & 6 months & 0.003899 & 0.2249 & 251 & 0.02 & 0.9862 \\
\hline
\end{tabular}

Table 5: Lower 50\% pairwise comparison of mean BMIs at all-time points post-operatively.

\begin{tabular}{|c|c|c|c|c|c|c|}
\hline Time of Visit (post-op) & Time of Visit (post-op) & Estimate & Standard Error & DF & t Value & $\operatorname{Pr}>|t|$ \\
\hline 1 month & 1 week & 0.05848 & 0.1412 & 116 & 0.41 & 0.6796 \\
\hline 1 month & 12 months & -0.2338 & 0.2013 & 116 & -1.16 & 0.2478 \\
\hline 1 month & 3 months & -0.09282 & 0.1570 & 116 & -0.59 & 0.5554 \\
\hline 1 month & 6 months & -0.2740 & 0.1783 & 116 & -1.54 & 0.1270 \\
\hline 1 week & 12 months & -0.2922 & 0.1969 & 116 & -1.48 & 0.1405 \\
\hline 1 week & 3 months & -0.1513 & 0.1527 & 116 & -0.99 & 0.3238 \\
\hline 1 week & 6 months & -0.3325 & 0.1755 & 116 & -1.89 & 0.0607 \\
\hline 12 months & 3 months & 0.1409 & 0.2045 & 116 & 0.69 & 0.4921 \\
\hline 12 months & 6 months & -0.04024 & 0.2189 & 116 & -0.18 & 0.8545 \\
\hline 3 months & 6 months & -0.1812 & 0.1839 & 116 & -0.99 & 0.3265 \\
\hline
\end{tabular}


Table 6: Upper 50\% pairwise comparison of mean BMls at all-time points post-operatively.

\begin{tabular}{|c|c|c|c|c|c|c|}
\hline Time of Visit (post-op) & Time of Visit (post-op) & Estimate & Standard Error & DF & t Value & $\operatorname{Pr}>|t|$ \\
\hline 1 month & 1 week & 0.03650 & 0.3318 & 131 & 0.11 & 0.9126 \\
\hline 1 month & 12 months & -0.1323 & 0.4165 & 131 & -0.32 & 0.7513 \\
\hline 1 month & 3 months & -0.3261 & 0.3567 & 131 & -0.91 & 0.3623 \\
\hline 1 month & 6 months & -0.1507 & 0.3795 & 131 & -0.40 & 0.6921 \\
\hline 1 week & 12 months & -0.1688 & 0.4072 & 131 & -0.41 & 0.6792 \\
\hline 1 week & 3 months & -0.3626 & 0.3439 & 131 & -1.05 & 0.2935 \\
\hline 1 week & 6 months & -0.1872 & 0.3705 & 131 & -0.51 & 0.6143 \\
\hline 12 month & 3 months & -0.1939 & 0.4229 & 131 & -0.46 & 0.6474 \\
\hline 12 months & 6 months & -0.01840 & 0.4344 & 131 & -0.04 & 0.9663 \\
\hline 3 months & 6 months & 0.1755 & 0.3898 & 131 & 0.45 & 0.6533 \\
\hline
\end{tabular}

( $22 \%$ of them increased BMI by at least $10 \%$ ). These authors observed that $24 \%$ of overweight patients at surgery (BMI 25.0$29.9 \mathrm{~kg} / \mathrm{m}^{2}$ ) dropped post-operatively to the "healthy BMI" category $\left(18.5-24.9 \mathrm{~kg} / \mathrm{m}^{2}\right)$, and $38 \%$ of obese patients (BMI $\geq 30$ $\mathrm{kg} / \mathrm{m}^{2}$ ) migrated down to the overweight category, with only $8 \%$ and $12 \%$ of patients changing BMI categories in the opposite direction, respectively [11]. As such, we also tiered the groups into the lowest $50 \%$ of patients based on baseline BMI was and the highest $50 \%$ of patients based on BMI. In the lower cohort, the 1 week post-operate mean BMI was 27.4589 (Table 2). This value was not statistically significantly higher than the mean BMI values at all other time points, specifically 1 month (BMI 27.4042), 3 months (BMI 27.4854), 6 months (BMI 27.9292), 1 year (BMI 28.1025), 2 years (BMI 28.4694) and greater than 2 years (BMI 28.5031). Table 5 illustrates no pairwise significance with any two time points and BMI within the lower $50 \%$ BMI cohort. The 1 week post-operative BMI for the higher $50 \%$ cohort was 36.5017 (Table 3). Compared to the time intervals at 1 month (BMI 36.6908), 3 months (BMI 37.0383), 6 months (BMI 36.2928), 1 year (BMI 37.2088), 2 years (BMI 37.1951) and greater than 2 years (BMI $36.8747)$, this value was not statistically significant. Again, there is no pairwise significant difference between any two time points for the higher 50\% BMI cohort (Table 6). In contrast to the work by Pike et al, there was no clinical (or statistical) weight gain with time for this cohort.

Coriddi et al. reported that obese patients who underwent reduction mammaplasty with the intention of losing weight ultimately required bariatric surgery to achieve their weight loss goals [12]. Interestingly, Singh et al. divided their study participants in two groups based on whether or not patients perceived the upcoming surgery as a stimulus for future weight loss, and later found significant postoperative weight loss only in the group who did [3] We did not preoperatively screen our patients' motivation, but it could quite possibly be the most important factor towards meaningful weight loss in macromastia patients, with reduction mammaplasty taking an enabling role. Further studies in this direction are needed.

A shortcoming of the current analyses is the relatively small sample size, although to our knowledge this is the largest series of objective adult data in this population. Additionally, there is more missing BMI data for patients farther away from the date of surgery, specifically for patients who have the lowest BMI. This is likely due to the lack of follow-up in eRecord for healthy patients. Our method of collecting BMI data through eRecord is through all encounters where a weight was obtained, not just within Plastic Surgery. Therefore, patients with higher BMIs may have additional co-morbidities that warrant more frequent health care provider encounters for all reasons. Presumably healthier patients with lower BMIs, although still overnight (BMI 25.00-29.99) were lost to a relatively short follow-up period. Because of this nonignorable missingness pattern and the relatively small sample of patients for which complete data is present, we are not able to find significant changes in BMI over time. Additionally, other factors that affect weight loss were not analyzed, such as other medical co-morbidities or age, level of physical activity and diet. Women may have experienced improvements in their health and cardiovascular status, although their BMI remained unchanged. A prospective study, specifically with strict BMI collection, different co-morbidities and exercise regimens over time tracked would be useful. Inclusion of a control group (perhaps nonsurgical patients with macromastia), which our current study lacks, could also provide an interesting comparison of weight changes and BMI over time.

\section{Conclusion}

Reduction mammoplasty is not associated with a decrease in $\mathrm{BMI}$ in patients with symptomatic macromastia. There was also no significant increase in BMI. Further studies are needed to compare the operated population to the nonoperated population as well as monitoring other metrics of health following surgery. 


\section{References}

1 Güemes A, Pérez E, Sousa R, Gil I, Valcarreres MP, et al. (2016) Quality of Life and Alleviation of Symptoms After Breast Reduction for Macromastia in Obese Patients: Is Surgery Worth It? Aesthetic Plast Surg 40: 62-70.

2 O'Blenes CA, Delbridge CL, Miller BJ, Pantelis A, Morris SF, et al. (2006) Prospective study of outcomes after reduction mammaplasty: long-term follow-up. Plast Reconstr Surg 117: 351-358.

3 Singh KA, Pinell XA, Losken A (2010) Is reduction mammaplasty a stimulus for weight loss and improved quality of life? Ann Plast Surg 64: 585-587.

4 Miller BJ, Morris SF, Sigurdson LL, Bendor-Samuel RL, Brennan $M$, et al. (2005) Prospective study of outcomes after reduction mammaplasty. Plast Reconstr Surg 115: 1025-1031.

5 Singh KA, Losken A (2012) Additional benefits of reduction mammaplasty: a systematic review of the literature. Plast Reconstr Surg 129: 562-570.

6 Karabekmez FE (2014) Does reduction mammaplasty revert skeletal disturbances in the vertebral column of patients with macromastia? A preliminary study. Aesthetic Plast Surg 38: 104-112.
7 Berberoğlu Ö, Temel M, Türkmen A (2015) Effects of Reduction Mammaplasty Operations on the Spinal Column: Clinical and Radiological Response. Aesthetic Plast Surg 39: 514-252.

8 Uzun H, Bitik O, Baltu Y, Sönmez C, Kaymak OA (2015) The Effects of Reduction Mammaplasty on Serum Leptin Levels and Insulin Resistance. Int J Endocrinol.

9 Kececi Y, Sir E, Gungor M (2015) Patient-reported quality-of-life outcomes of breast reduction evaluated with generic questionnaires and the breast reduction assessed severity scale. Aesthet Surg J 35: 48-54.

10 Pauzenberger R, Bauer T, Kirchebner M, Pikula R, Verstappen R, et al. (2014) Reduction mammaplasty: outcome analysis based on bodyweight. Aesthetic Plast Surg 38: 120-128.

11 Pike CM, Nuzzi LC, DiVasta AD, Greene AK, Labow B (2015) Weight Changes After Reduction Mammaplasty in Adolescents. J Adolesc Health 57: 277-281.

12 Coriddi M, Koltz PF, Gusenoff JA (2011) Reduction mammaplasty, obesity, and massive weight loss: temporal relationships of satisfaction with breast contour. Plast Reconstr Surg 128: 643-650. 